\title{
Increased Oxidant Stress and Inflammation in Patients with Chronic Schizophrenia
}

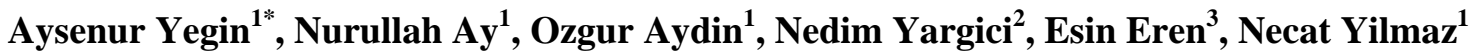 \\ ${ }^{1}$ Central Laboratory, Department of Biochemistry, Antalya Educational and Research Hospital, Antalya, Turkey; ${ }^{2}$ Department of \\ Psychiatry, Antalya Educational and Research Hospital, Antalya, Turkey; ${ }^{3}$ Central Laboratory, Ataturk Hospital of Ministry of \\ Health, Antalya, Turkey. \\ Email: *aysenuryegin@yahoo.com
}

Received June $22^{\text {nd }}, 2012$; revised July $24^{\text {th }}, 2012$; accepted August $6^{\text {th }}, 2012$

\begin{abstract}
Background: Several lines of evidence, including postmortem studies, suggest increased oxidative stress and inflammation in patients with schizophrenia. Alteration of oxidative stress markers has been reported in schizoprenia studies, but with inconsistent results. Oxidized low-density lipoproteins (oxLDL) have been reported to be capable of eliciting neurocytotoxicity. On the other hand, paraoxonase (PON1), an arylesterase(ARE), plays a role in protection against oxidative modifications of LDL and is considered to be one of the antioxidant enzymes. There are no studies showing the changes in oxidative stress and inflammation together, nor the activities of PON1 and ARE in schizophrenic patients. In this study, we examined PON1, ARE activities and oxidative/anti-oxidative markers in patients with chronic schizophrenia and healthy controls. Methods: We recruited 30 male chronic schizophrenic patients and 30 male healthy control subjects and examined C-reactive protein(CRP), fibrinogen, PON1, ARE and plasma total antioxidant status (TAS) and total oxidant status (TOS), oxidative stress index(OSI) in both groups. Schizophrenia symptoms were assessed using the positive and negative syndrome scale (PANSS). The related routine lipid profile parameters including HDL were also examined. Results: Patients had significantly higher CRP, fibrinogen, TOS and OSI levels; but the patients and control subjects did not differ on activities of the antioxidant enzymes PON1 and ARE. Interestingly, there were not any group differences in the lipid profile parameters except the triglyceride levels, that increased significantly in the patient group. Conclusions: In the present study, reporting the ARE activities besides the PON1 activities in schizophrenic patients for the first time, we showed that PON1 and ARE enzyme activities were not statistically different in patients with chronic schizophrenia. This study provides additional evidence of increased oxidative stress and inflammation in chronic schizophrenia, but no alterations in the antioxidant status were observed. Our results suggest that other mechanisms than the high density lipoprotein(HDL)-disfunctionality, namely decreases in PON1 or ARE enzyme activities, are more important in oxidative or antioxidative pathophysiological processes in schizophrenia.
\end{abstract}

Keywords: Oxidative Stress; Antioxidant Status; Inflammation; Paraoxonase; Schizophrenia

\section{Introduction}

"Schizophrenia is still one of the most mysterious and costliest mental disorders in terms of human suffering and societal expenditure" [1]. Accumulating evidence points to many interrelated mechanisms that increase production of reactive oxygen or decrease antioxidant protection in schizophrenic patients [2]. However, the reports regarding the status of oxidative stress markers in schizophrenia are very inconsistent, with various authors stating both increased and decreased activities of the main antioxidant enzymes, while others did not observe any significant modifications, as compared to control

${ }^{*}$ Corresponding author. groups [3].

The chief cause of excess premature mortality among patients with schizophrenia is cardiovascular heart disease, caused mainly by their adverse risk factor profile [4]. Schizophrenia patients have a higher chance (prevalence of $36 \%$ ) of developing metabolic syndrome, even without antipsychotic medication [5]. Not only diabetes mellitus but multiple risk factors for cardiovascular diseases are significantly increased in this patient group [6]. Schizophrenic patients have a higher risk of raised cholesterol/HDL ratio, and also smoke more often. Some risk factors are already present at the onset of the psychotic disorder [5]. Low levels of HDL are typical of the biochemical cluster defining metabolic 
syndrome. Disturbances in the concentrations of apoproteins, function of enzymes, transport proteins, receptors, other lipoproteins, and their clearance from plasma can have a major impact on the anti-atherogenic properties in HDL. Furthermore, HDLs are one of the most important antioxidant defence systems in plasma. They are well known to prevent LDL oxidation and protect against LDL-induced cytotoxicity [7-10]. HDLs also possess anti-inflammatory properties, including the ability of suppressing cytokine-induced endothelial cell adhesion molecules function [11-13].

The antioxidant properties of HDLs are, at least to some extent, attributable to serum PON1. PON1, an ARE, plays a role in protection against oxidative modifications of LDL and is considered to be one of the antioxidant enzymes [14]. Taken together, these data suggest that PON1, an antioxidant enzyme, may be involved in the pathophysiology of schizophrenia. The present study was therefore undertaken to investigate the PON1 and ARE activities besides the oxidant stress index and inflammation markers, in a cohort of patients diagnosed as chronic schizophrenia.

\section{Materials and Methods}

\subsection{Subjects}

Blood from 30 selected male patients with chronic schizophrenia and 30 normal healthy age matched male controls was assayed in this study. The patients were enrolled from the Psychiatric Clinic, Research and Education Hospital, Antalya, Turkey. Healthy controls were recruited from the hospital staff and were also assessed by a semistructured psychiatric interview. The study was approved by the local ethics committee. The trial procedure was in accordance with the guidelines of 2002 Declaration of Helsinki.

A Diagnostic and Statistical Manual of Mental Disorders (DSM-IV) diagnosis of chronic schizophrenia was established on the basis of independent clinical interviews; and reviews of the patient records by two psychiatrists using the Brief Psychiatric Rating Scale (BPRS), the Scale for the Assessment of Negative Symptoms (SANS), and the Scale for the Assessment of Positive Symptoms (SAPS) [15]. Study criteria for patients with schizophrenia were as follows: 1) diagnosis of schizophrenia according to Diagnostic and Statistical Manual (DSMIV); 2) no other DSM-IV axis I diagnosis; 3) no history of alcohol or substance abuse or dependence; 4) absence of antioxidant administration for at least one year; 5) no concomitant or past severe medical conditions; 6) nonsmoking; 7) ability to provide informed consent. All patients presented the typical symptoms of schizophrenia, that were divided into positive and negative, through the defined guidelines. Positive symptoms were those that appeared to reflect an excess or distortion of normal functions, like delusions, hallucinations, disorganized speech and thinking, grossly disorganized behavior, catatonic behaviors. Negative symptoms were those that appeared to reflect a diminution or loss of normal functions, like affective flattening, alogia, and avolition. The absence of medical or neurological illness was verified by means of physical and neurological examination, routine laboratory investigation, treating physician reports, and medical records. Individuals without any clearly evident psychiatric illness or substance abuse were recruited as control subjects for the study. Study criteria for healthy controls were: 1) absence of past or present neurological or psychiatric illnesses; 2) absence of concomitant or past severe medical conditions; and 3) informed consent. Healthy controls were group matched to patients for age, gender and race.

The patients were treated with various antipsychotic drugs (>60 months), 19 with second generation antipsychotics (nine with risperidone, five with olanzapine, five with clozapine) and 11 with classic antipsychotics (haloperidol).

\subsection{Analytical Methods}

\subsubsection{Blood Sample Collection}

Blood samples were obtained after an overnight fasting state. Serum samples were then separated from the cells by centrifugation at $3000 \mathrm{rpm}$ for 10 minutes, and lipid parameters were measured freshly. Remaining serum portions were stored at $-80^{\circ} \mathrm{C}$ and used to analyze PON1 and ARE enzyme activities and TOS and TAS levels.

\subsubsection{Measurement of Paraoxonase and Arylesterase Activities of Serum}

PON1 and ARE activities were measured using comercially available kits (Relassay ${ }^{\circledR}$, Turkey). Fully automated PON1 activity measurement method consists of two different sequential reagents. The first reagent is an appropriate Tris buffer and it also contains calcium ion, which is a cofactor of PON1 enzyme. Linear increase of the absorbance of $p$-nitrophenol, produced from paraoxon, is followed at kinetic measurement mode. Nonenzymatic hydrolysis of paraoxon was substracted from the total rate of hydrolysis. The molar absorptivity of $p$-nitrophenol is $18,290 \mathrm{M}^{-1} \cdot \mathrm{cm}^{-1}$ and one unit of paraoxonase activity is equal to $1 \mathrm{~mol}$ of paraoxon hydrolyzed per liter per minute at $37^{\circ} \mathrm{C}[16]$.

Phenylacetate was used as a substrate to measure the ARE activity. PON1, present in the sample, hydrolyses phenylacetate to its products, which are phenol and acetic acid. The produced phenol is colorimetrically measured via oxidative coupling with 4-aminoantipyrine and potassium ferricyanide. Nonenzymatic hydrolysis of phenyl 
acetate was subtracted from the total rate of hydrolysis. The molar absorptivity of colored complex is 4000 $\mathrm{M}^{-1} \cdot \mathrm{cm}^{-1}$ and one unit of arylesterase activity is equal to $1 \mathrm{mmol}$ of phenylacetate hydrolyzed per liter per minute at $37^{\circ} \mathrm{C}[17]$.

\subsubsection{Measurement of the Total Antioxidant Status of Serum}

The TAS of the serum was measured using a novel automated colorimetric measurement method for TAS developed by Erel [18]. In TAS method, antioxidants in the sample reduce dark blue-green colored 2,2'-azinobis (3-ethylbenzthiazoline-6-sulphonic acid) (ABTS) radical to colorless reduced ABTS form. The change of absorbance at $660 \mathrm{~nm}$ is related with total antioxidant level of the sample. Using this method, the antioxidative effect of the sample against the potent free radical reactions initiated by the produced hydroxyl radical, is measured. The results are expressed as micromolar trolox equivalent per liter.

\subsubsection{Measurement of the Total Oxidant Status of Serum}

The TOS of the plasma was measured using a novel automated colorimetric measurement method for TOS developed by Erel [19]. In TOS method; oxidants present in the sample oxidize the ferrous ion-chelator complex to ferric ion. The ferric ion makes a colored complex with chromogen in an acidic medium. The color intensity, which can be measured spectrophotometrically, is related to the total amount of oxidant molecules present in the sample. The results are expressed in terms of micromolar hydrogen peroxide equivalent per liter $\left(\mu \mathrm{mol} \mathrm{H}_{2} \mathrm{O}_{2}\right.$ Equiv. /L).

\subsubsection{Oxidative Stress Index}

The percentage ratio of TOS level to TAS level was accepted as oxidative stress index (OSI) [20]. For calculation, the resulting unit of TAS was changed to millimoles per liter, and the OSI value was calculated according to the following formula: OSI (arbitrary unit) $=$ TOS (micromolar hydrogen peroxide equivalent per liter)/TAS (micromolar trolox equivalent per liter) [19].

\subsubsection{Routine Parameters}

The levels of triglycerides (TG), total cholesterol (TC), high-density lipoprotein cholesterol (HDL-C), and Lowdensity lipoprotein cholesterol (LDL-C) were determined by using commercially available assay kits (Abbott) with an autoanalyzer (Architect ${ }^{\circledR}$ c16000, Abbott Diagnostics). A nephelometric method was used for measuring CRP levels (Delta Array ${ }^{\circledR}$ Protein System, SEAC Diagnostics) and fibrinogen was measured with a coagulometer (Trombolyzer ${ }^{\mathbb{R}}$ XRC, Behnk Elektronik). Vitamin B12 and folate levels were determined by using the in- strument Beckman Coulter DXI 800. The Access Vitamin B12 assay was a competitive-binding immunoenzymatic assay and the Access Folate assay was a competetive-binding receptor assay. Non-HDL-C levels were calculated using the formula: Non-HDL-C Level $=$ Total Cholesterol Level-HDL-C Level.

\subsection{Statistical Analysis}

Statistical analyses were carried out using the MedCalc statistical software (MedCalc, Mariakerke, Belgium). The results were expressed as mean $\pm \mathrm{SD}$. The significance of the differences between groups was determined by student's unpaired $t$-test and the Wilcoxon test. Pearson correlation coefficient was used to test the strength of any associations between different variables. $\mathrm{P}$ values less than 0.05 was accepted as the significance level. The c statistic was used to observe the optimal cut-off levels and associated diagnostic performances (sensivity, specificity, and diagnostic value) of PON, ARE, TAS, TOS, and OSI, based on area under the receiver operating characteristic (ROC) curve (AUC) analysis.

\section{Results}

Clinical data of patients and age-matched controls are summarized in Table 1. The means of BMI of the groups did not differ significantly. There were no differences between cases and controls with regard to body mass index and age.

Mean of total cholesterol (TC) in schizophrenic patients was $187.8 \pm 43.3 \mathrm{mg} / \mathrm{dL}$ which was not statistically any different compared to the control subjects (177.5 \pm $32.4 \mathrm{mg} / \mathrm{dL}$ ) as shown in Table 2. Similarly, neither of means of serum HDL-C and serum LDL-C were signifycantly different between the patients and the controls (Table 2). However, mean of serum TG in patients $(180.6 \pm 85.3 \mathrm{mg} / \mathrm{dL})$ was significantly higher compared to the controls $(109.7 \pm 51.4 \mathrm{mg} / \mathrm{dL})(\mathrm{p}=0.0003)$ (Table 2). Patients did not differ from controls in terms of the nutritionalparameters, plasma folate and vitamin B12 levels. On the contrary, the inflammation markers CRP and fibrinogen were higher in the patient group (Table 2).

Table 1. Sample demographics.

\begin{tabular}{lccc}
\hline Parameter $($ mean $\pm \mathrm{SD})$ & Patients $(\mathrm{n}=30)$ & Controls $(\mathrm{n}=30)$ & $\mathrm{P}$ \\
\hline Age & $36.7 \pm 9.4$ & $38.3 \pm 15.3$ & 0.643 \\
Weight & $73.4 \pm 10$ & $74 \pm 9.7$ & 0.65 \\
Gender & All males & All males & \\
BMI $\left(\mathrm{kg} / \mathrm{m}^{2}\right)$, mean $\pm \mathrm{SD}$ & $25.54 \pm 2.54$ & $25.15 \pm 4.0$ & 0.646 \\
BPRS total score & $18.79 \pm 9.30$ & - & \\
SANS total scores & $57.87 \pm 27.18$ & - & \\
SAPS total scores & $34.00 \pm 29.75$ & - & \\
\hline
\end{tabular}


We also investigated the serum TAS, TOS and OSI levels of schizophrenia patients and the controls. We found TOS and OSI higher in patients compared to controls, but no significant difference was observed in TAS levels. PON1 and ARE enzyme activities were not either different between the patients and the control group. The results are presented in Table 3. Table 4 shows the significant correlation between OSI and CRP.

Optimal cut-off levels and associated diagnostic performances (sensivity, specificity, and diagnostic value) of PON, ARE, TAS, TOS, and OSI, based on ROC analysis, are given in Table 5. We presented the ROC

Table 2. Blood lipid, inflammation and nutritional parameters in schizophrenia patients and controls.

\begin{tabular}{lccc}
\hline $\begin{array}{l}\text { Serum Parameters } \\
(\mathrm{mean} \pm \mathrm{SD})\end{array}$ & $\begin{array}{c}\text { Patients } \\
(\mathrm{n}=30)\end{array}$ & $\begin{array}{c}\text { Controls } \\
(\mathrm{n}=30)\end{array}$ & P values \\
\hline Total Cholesterol (mg/dL) & $187.8 \pm 43.3$ & $177.5 \pm 32.4$ & 0.302 \\
Triglyceride $(\mathrm{mg} / \mathrm{dL})$ & $180.6 \pm 85.3$ & $109.7 \pm 51.4$ & 0.0003 \\
HDL Cholesterol $(\mathrm{mg} / \mathrm{dL})$ & $41.5 \pm 10.4$ & $45.9 \pm 10.7$ & 0.107 \\
LDL Cholesterol (mg/dL) & $110.1 \pm 41.0$ & $109.5 \pm 31.3$ & 0.950 \\
$\begin{array}{l}\text { Non-HDL Cholesterol } \\
(\mathrm{mg} / \mathrm{dL})\end{array}$ & $146 \pm 42$ & $131 \pm 32$ & 0.13 \\
Folic Acid (ng/mL) & $5.31 \pm 2.31$ & $5.94 \pm 1.9$ & 0.21 \\
Vitamin B12 (pg/mL) & $182 \pm 83$ & $152 \pm 62$ & 0.12 \\
Fibrinogen (mg/dL) & $358.9 \pm 121$ & $292.4 \pm 97$ & 0.0094 \\
CRP ${ }^{*}(\mathrm{mg} / \mathrm{L})$ & $5.0987 \pm 4.74^{*}$ & $2.84 \pm 1.03$ & 0.017 \\
\hline
\end{tabular}

*Wilcoxon test.

Table 3. Serum PON1, ARE, TAS, TOS and OSI levels of schizophrenia patients compared to the controls.

\begin{tabular}{lccc}
\hline Parameter $($ mean $\pm \mathrm{SD})$ & Patients $(\mathrm{n}=30)$ & Controls $(\mathrm{n}=30)$ & $\mathrm{P}$ \\
\hline PON1(U/L) & $99.9 \pm 61.6$ & $93.7 \pm 44.5$ & 0.660 \\
ARE $(\mathrm{kU} / \mathrm{L})$ & $150.1 \pm 59.3$ & $163.8 \pm 66.1$ & 0.402 \\
TAS $(\mathrm{nmol}$ Troloks/L) & $1.44 \pm 0.33$ & $1.52 \pm 0.30$ & 0.327 \\
TOS & $67.4 \pm 60.1$ & $8.69 \pm 2.91$ & 0.0001 \\
$\left(\mu \mathrm{mol} \mathrm{H}_{2} \mathrm{O}_{2}\right.$ Equiv./L) & & & \\
OSI & $12297.3 \pm 3963.8$ & $564.7 \pm 121.3$ & 0.0001 \\
PON/ARE $^{*}$ & $0.66 \pm 0.29$ & $0.61 \pm 0.27$ & 0.51 \\
\hline
\end{tabular}

*Wilcoxon test. curves for all the parameters in Figure 1. The sensitivity and specificity of TOS were $80 \%$ and $93.3 \%$, respectively, and the AUC was 0.893 (Figure 2). According to the ROC curve for OSI, the diagnostic sensitivity and specificity were $80.0 \%$ and $93.3 \%$, respectively, and the AUC was 0.878 (Figure 3). The AUC for the PON and ARE were 0.504 and 0.549 respectively, and these values were lower than that of TOS and OSI.

\section{Discussion}

Since over a century that schizophrenia has been conceptualized there have been wide-ranging variety of pathophysiological models and causal hypotheses of schizophrenia [21]. One among these is the role for free radical-mediated pathology in schizophrenia that was proposed more than half century ago [22]. Brain is particularly vulnerable to oxidative stress as a result of the relatively low levels of antioxidants, high levels of

Table 4. Correlation Coefficients in schizoprenia group.

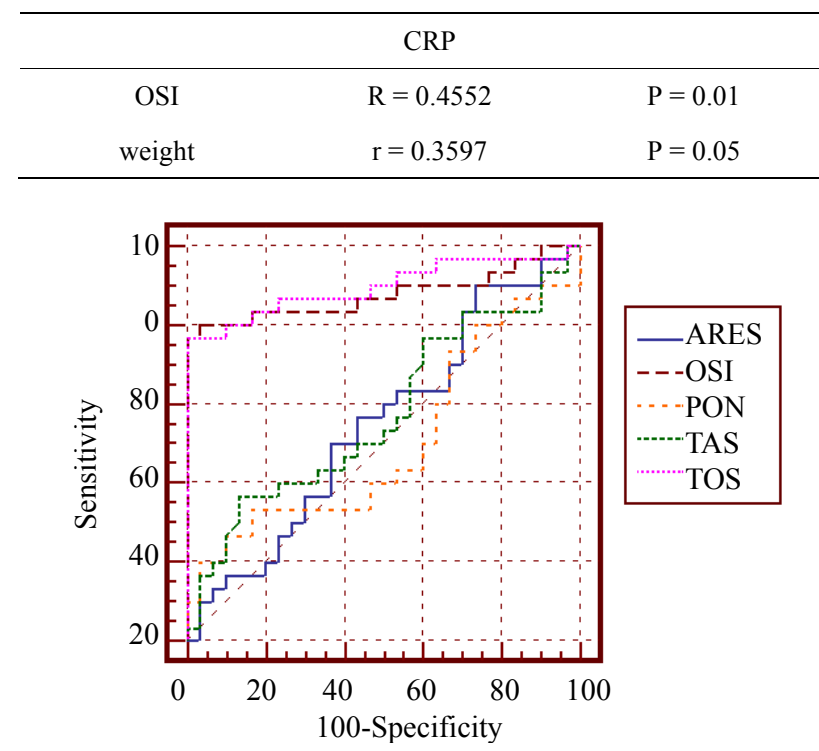

Figure 1. The figure compares the diagnostic performances (sensitivity and specificity) of PON, ARE, TAS, TOS and OSI based on ROC analysis.

Table 5. Optimal cut-off levels and associated diagnostic performances (sensitivity, specificity, and diagnostic value) of PON, ARE, TAS, TOS and OSI based on ROC analysis, are given in the table. The AUC for the PON, and ARE were 0.504 and 0.549 , respectively, and these values were lower than those of TOS $(0.897)$ and OSI (0.878).

\begin{tabular}{ccccccc}
\hline Biomarker & Cut-off level & Sensitivity (\%) & Specificity (\%) & Diagnostic value (AUC) & + LR & - LR \\
\hline PON1 & 47.0 & 26.7 & 90.0 & 0.504 & 2.67 & 0.81 \\
ARE & 204.3 & 90.0 & 26.7 & 0.549 & 1.23 & 0.37 \\
TAS & 1.2 & 36.7 & 86.7 & 0.576 & 2.75 & 0.73 \\
TOS & 15.0 & 76.7 & 93.3 & 0.897 & 11.50 & 0.25 \\
OSI & 788.1 & 80.0 & 93.3 & 0.878 & 24.00 & 0.21 \\
\hline
\end{tabular}

$+\mathrm{LR}=$ Positive likelihood ratio; $-\mathrm{LR}=$ Negative likelihood ratio. 


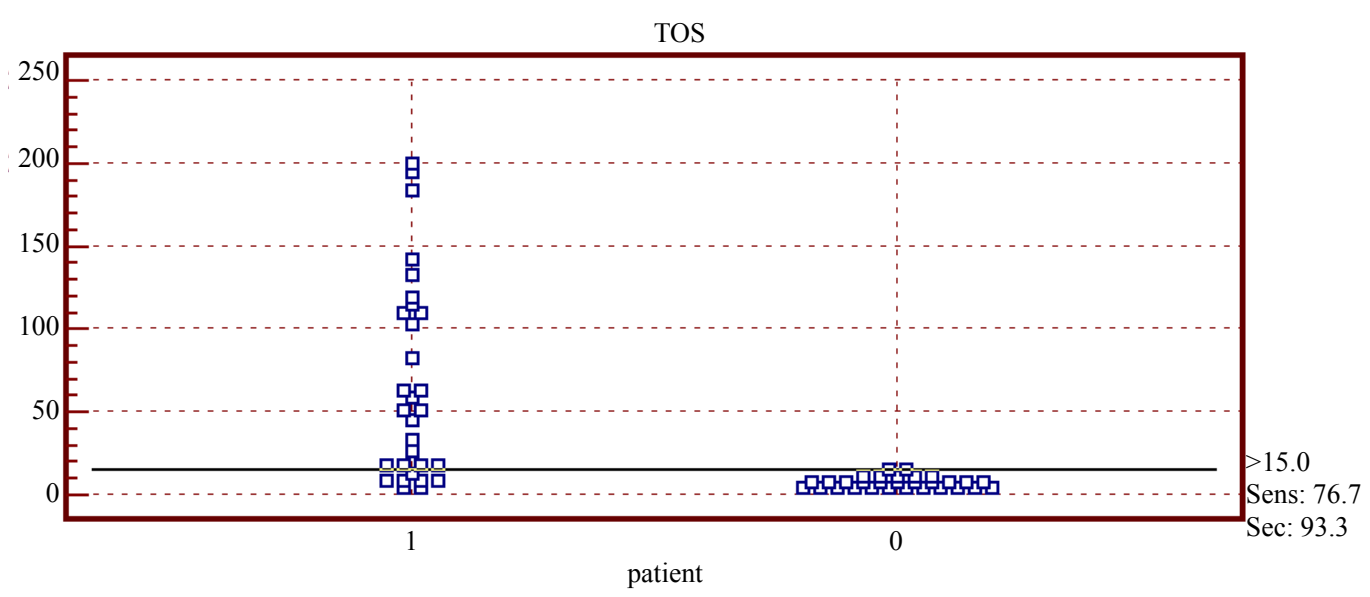

Figure 2. The sensitivity and specificity of TOS were $76.7 \%$ and $93.3 \%$, respectively, and the AUC was 0.897 (1: schizophrenia patients; 0: controls).

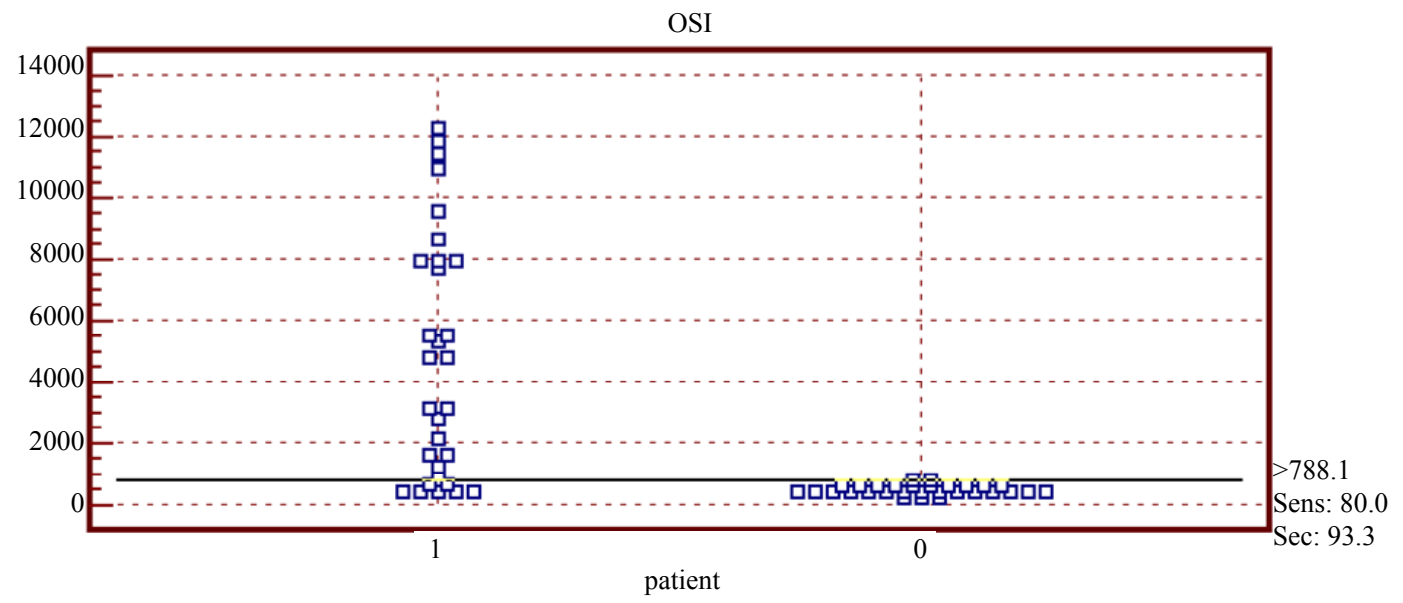

Figure 3. The sensitivity and specificity of OSI were $80.0 \%$ and $93.3 \%$, respectively, and the AUC was 0.878 (1: schizophrenia patients; 0: controls).

polyunsaturated fatty acids and increased need of oxygen $[23,24]$. In the intervening decades there has been a steady stream of evidence demonstrating the presence of oxidative stress in patients with schizophrenia [21]. In accordance with these implications, our results showed that TOS and OSI increased in our patient group compared to the controls, providing additional evidence of increased oxidative stress in schizophrenia (Tables 3 and 5). Moreover, ROC analysis revealed high diagnostic values for TOS and OSI with respect to male patients with schizophrenia, with an area under curve (AUC) of 0.897 and 0.878 , respectively as shown in the Figures 1-3.

Additionally, our results for CRP, a prototypic marker of inflammation, and fibrinogen suggest that inflammation is enhanced in the schizophrenic patients in our study group (Table 2). Fibrin (ogen) has been shown to cause an inflammatory response in peripheral blood mononuclear cells induced by high levels of reactive oxygen species, increased cytokine and chemokine ex- pression and macrophage chemoattractant protein-1 [25]. In their review, Leonard et al. stated that inflammatory mediators including CRP and fibrinogen, are raised in the serum of patients with schizophrenia. Thus they concluded that there is a chronic, low-grade inflammatory change associated with the active phase of schizophrenia [26]. Oxidative stress and inflammation are inextricably tied processes. Chronic inflammation is associated with elevated reactive oxygen species levels; anti-inflammatory cascades are linked to diminished reactive oxygen species concentrations. And the converse is trueelevated oxidative stress triggers inflammation, whereas redox balance inhibits the cellular response. Thus, oxidative stress and inflammation may be seen as both causes and consequences of cellular pathology in many disorders [27], probably in degenarative diseases such as schizophrenia.

Schizophrenic patients are in general expected to be an undernourished group and, as a consequence, may show low vitamin levels; however there was no significant 
difference in folate and B12 levels between the patient and control groups in this study (Table 2). In agreement with our findings, other authors reported that schizophrenic patients in their study groups also had folate and B12 levels within normal limits [28]. These findings suggest that the patients in our study group were not undernourished.

The potential toxicity of free radicals is counteracted by a number of cytoprotective antioxidant enzymes that limit the damage, such as superoxide dismutase and glutathione peroxidase. However, the reports regarding the status of antioxidant defence system in schizophrenia are very inconsistent, with various authors stating both increased and decreased activities of the main antioxidant enzymes, while others did not observe any significant modifications, as compared to control groups [29]. Studies performed in schizophrenia patients have generally suggested a compromised antioxidant system, but this is not always consistent with specific observed parameters, which on the whole, show evidences of dysregulation. Reduced levels of the antioxidant enzymes are generally reported in patients with schizophrenia compared with controls [30-33]. However, some studies have advocated a strengthening of antioxidant status in schizophrenia [34-37]. In our study, we observed no significant difference in TAS values of our study groups. As our understanding of the interrelations of the components of the antioxidant defence system enlarges, it becomes obvious that examining one enzyme in isolation may have limited value for elucidating the role of impaired antioxidant defence system in neuropsychiatric disease processes, suggesting that the dynamic aspects of the antioxidant defence system likely have more salience to understanding the pathophysiology of schizophrenia [38]. It is generally believed that the equivocal results mentioned above may be due to different tissue studies, different species or the administrated treatment and the duration of the disease/treatment [3]. On the other hand, changes in antioxidant defense system such as decreased plasma TAS do not necessarily reflect an increased oxidative stress and subsequent membrane lipid damage [29].

Patients with major mental illnesses such as schizophrenia and bipolar disorder have increased risks of morbidity and mortality compared with the general population, with a 25- to 30-year shorter life span due primarily to premature cardiovascular disease [39]. Oxidative stress, owing to increased lipid and protein oxidation products, is associated with cardiovascular diseases and affects PON1 expression and activities [40]. Although the primary physiological role of PON1 is still uncertain, a recent study has shown that PON1 is located on HDL and plays a role in protection against oxidative modification of LDL; that is, lipid peroxidation [41].
PON1 enzyme activity is associated with HDL functionality and it is reported to be responsible for the antioxidant properties of HDL [42]. In the present study, we showed that PON1 and ARE enzyme activities were not statistically different in patients with chronic schizophrenia. To our knowledge, this is the first paper reporting the ARE activities besides the PON1 activities in schizophrenic patients. Although PON1 and ARE are considered to be two different enzymes, previous studies have shown that a single gene product in human serum has both ARE and PON1 activities [43]. PON1 is located on $\mathrm{Chr} 7 \mathrm{q} 21.3$ and the Gln192Arg polymorphism is associated with PON1 activity to protect LDL against oxidative modification [14]. Matsumoto et al. [14] did not observe a significant association between schizophrenia and one nonsynonymous polymorphism (Gln192Arg). Comparison of the ability of HDL to protect LDL from oxidation between PON1 192Gln/Gln genotype and PON1 $192 \mathrm{Arg} / \mathrm{Arg}$ genotype demonstrated that the $192 \mathrm{Gln} / \mathrm{Gln}$ genotype exhibited approximately 6-fold higher efficiency than the 192Arg/Arg genotype [44].

The only lipid profile parameter that significantly differed from those of the control subjects was the serum triglyceride levels in our patient group (Table 2). On the other hand, it was interesting to observe that HDLcholesterol levels seemed to be lower, but not significantly different in the shizophrenic patients compared to the control subjects (Table 2). In the context of high triglycerides, low HDL cholesterol is not only an early symptom, but also provides a very sensitive marker of impaired glucose tolerance and increased lipolysis. These examples reflect the very heterogeneous nature of HDL which makes HDL genetics very complex [45]. Although high-density lipoprotein-cholesterol levels in large epidemiological studies are inversely related to the risk of coronary heart disease (CHD), increasing the level of circulating HDL does not necessarily decrease the risk of CHD events, CHD deaths, or mortality [46]. Typically, total plasma HDL contains $20 \%-30 \%(\mathrm{wt} / \mathrm{wt})$ phospholipids, $3 \%-5 \%$ free cholesterol, $14 \%-18 \%$ cholesteryl ester and 3\% - $6 \%$ triglycerides. Another factor that might make HDL dysfunctional is a change in the HDL associated lipids. Enrichment in triglyceride with depletion of cholesteryl ester in the HDL core is the most frequent abnormality of HDL lipid composition and occurs in hypertriglyceridemic states associated with decreased activity of lipoprotein lipase, hepatic lipase, LCAT, or a combination of these. In addition, HDL triglyceride content can also be increased in hypertriglyceridemia as a consequence of elevated cholesteryl ester transfer protein activity [47]. Because low plasma HDL concentration sometimes is associated with increased risk of cardiovascular diseases, whereas other conditions with low plasma HDL concentration are as- 
sociated with improved prognosis, it seems that it is not only the concentration per se but also the function of the HDL particles that is important for its antiatherogenic effects. HDL particles are susceptible to structural modifications mediated by various mechanisms, including oxidation, glycation, or enzymatic degradation, affecting their functional properties. Moreover, in vitro studies have shown that homocysteinylation of HDL may reduce the activity of the enzyme PON1, which is associated with human HDL, thus rendering the HDL particle more susceptible to oxidative damage $[48,49]$.

All the patients in our patient group were under treatment with various antipsychotic drugs. In the previous studies, it was shown that neither individual antioxidant levels nor total antioxidant status were significantly affected by antipsychotic treatment [25,50-52]. More importantly, the altered antioxidants observed were independent of treatment since patients were antipsychotic drug-naive at entry into the study [29,50,51]. Therefore, we could suggest that the antioxidant enzyme activities of PON1 and ARE were not affected by the antipsychotic drugs used to treat our patient group.

\section{Conclusion}

This study provides additional evidence of increased oxidative stress and inflammation in chronic schizophrenia, but no alterations in the antioxidant status nor in the enzymatic activities of PON1 and ARE were observed. Our results suggest that other mechanisms than the HDL-disfunctionality, namely decreases in PON1 or ARE enzyme activities, are more important in oxidative or antioxidative pathophysiological processes in schizophrenia.

\section{REFERENCES}

[1] J. van Os and S. Kapur, "Schizophrenia," Lancet, Vol. 22, No. 374, 2009, pp. 635-645. doi:10.1016/S0140-6736(09)60995-8

[2] C. Fendri, A. Mechri, G. Khiari, A. Othman, A. Kerkeni and L. Gaha, "Oxidative Stress Involvement in Schizophrenia Pathophysiology: A Review," L'Encéphale, Vol. 32, No. 2, 2006, pp. 244-252. doi:10.1016/S0013-7006(06)76151-6

[3] A. Ciobica, M. Padurariu, I. Dobrin, C. Stefanescu and R. Dobrin, "Oxidative Stress in Schizophrenia-Focusing on the Main Markers," Psychiatria Danubina, Vol. 23, No. 3, 2011, pp. 237-245.

[4] C. H. Hennekens, A. R. Hennekens, D. Hollar and D. E. Casey, "Schizophrenia and Increased Risks of Cardiovascular Disease," American Heart Journal, Vol. 150, No. 6, 2005, pp. 1115-1121. doi:10.1016/j.ahj.2005.02.007

[5] M. J. T. Oud and B. M.-de Jong, "Somatic Diseases in Patients with Schizophrenia in General Practice: Their Prevalence and Health Care," BMC Family Practice, Vol.
10, 2009, p. 32. doi:10.1186/1471-2296-10-32

[6] D. P. J. Osborn, I. Nazareth and M. B. King, "Risk for Coronary Heart Disease in People with Severe Mental İllness. Cross-Sectional Comparative Study in Primary Care," The British Journal of Psychiatry, Vol. 188, 2006, pp. 271-277. doi:10.1192/bjp.bp.104.008060

[7] S. Parthasarathy, J. Barnett and L. G. Fong, "HighDensity Lipoprotein İnhibits the Oxidative Modification of Low-Density Lipoprotein," Biochimica et Biophysica Acta, Vol. 1044, No. 2, 1990, pp. 275-283. doi:10.1016/0005-2760(90)90314-N

[8] M. Navab, G. M. Ananthramaiah, S. T. Reddy, et al., "The Oxidation Hypothesis of Atherogenesis: The Role of Oxidized Phospholipids and HDL," Journal of Lipid Research, Vol. 45, No. 6, 2004, pp. 993-1007. doi:10.1194/jlr.R400001-JLR200

[9] M. Navab, S. Y. Hama, G. M. Anantharamaiah, et al., "Normal High Density Lipoprotein Inhibits Three Steps in the Formation of Mildly Oxidized Low Density Lipoprotein: Steps 2 and 3," Journal of Lipid Research, Vol. 41, No. 9, 2000, pp. 1495-1508.

[10] M. Navab, S. T. Reddy, B. J. Van Lenten, G. M. Anantharamaiah and A. M. Fogelman, "The Role of Dysfunctional HDL in Atherosclerosis," Journal of Lipid Research, Vol. 50, 2009, pp. S145-S149. doi:10.1194/j1r.R800036-JLR200

[11] G. W. Cockerill, K. A. Rye, J. R. Gamble, M. A. Vadas and P. J. Barter, "High-Density Lipoproteins Inhibit Cytokine-Induced Expression of Endothelial Cell Adhesion Molecules," Arteriosclerosis, Thrombosis, and Vascular Biology, Vol. 15, No. 11, 1995, pp. 1987-1994. doi:10.1161/01.ATV.15.11.1987

[12] L. Calabresi, M. Gomaraschi, B. Villa, L. Omoboni, C. Dmitrieff and G. Franceschini, "Elevated Soluble Cellular Adhesion Molecules in Subjects with Low HDL-Cholesterol," Arteriosclerosis, Thrombosis, and Vascular Biology, Vol. 22, No. 4, 2002, pp. 656-661. doi:10.1161/hq0402.105901

[13] G. W. Cockerill, T. Y. Huehns, A. Weerasinghe, et al., "Elevation of Plasma High-Density Lipoprotein Concentration Reduces İnterleukin-1-Induced Expression of E Selectin in an in Vivo Model of Acute Inflammation," Circulation, Vol. 103, No. 1, 2001, pp. 108-112. doi:10.1161/01.CIR.103.1.108

[14] C. Matsumoto, O. Ohmori, H. Hori, T. Shinkai and J. Nakamura, "Analysis of Association between the Gln192Arg Polymorphism of the Paraoxonase Gene and Schizophrenia in Humans," Neuroscience Letters, Vol. 321, No. 3, 2002, pp. 165-168. doi:10.1016/S0304-3940(02)00049-6

[15] S. R. Kay, A. Fiszbein and L. A. Opler, "The Positive and Negative Syndrome Scale (PANSS) for Schizophrenia," Schizophrenia Bulletin, Vol. 13, No. 2, 1987, pp. 261267.

[16] H. W. Eckerson, M. C. Wyte and B. N. La Du, "The Human Serum Paraoxonase/Arylesterase Polymorphism," The American Journal of Human Genetics, Vol. 35, No. 6, 1983, pp. 1126-1138.

[17] L. Haagen and A. Brock, "A Newautomatedmethod for 
Phenotyping Arylesterase (E.C.3.1.1.2.) Based upon İnhibition of Enzymatic Hydrolisis of 4-Nitrophenyl Acetate," European Journal of Clinical Chemistry and Clinical Biochemistry, Vol. 30, No. 7, 1992, pp. 391-395.

[18] O. Erel, "A Novel Automated Direct Measurement Method for Total Antioxidant Capacity Using a New Generationmore Stable ABTS Radical Cation," Clinical Biochemistry, Vol. 37, No. 4, 2004, pp. 277-285. doi:10.1016/j.clinbiochem.2003.11.015

[19] O. Erel, "A New Automated Colorimetric Method for Measuring Total Oxidant Status," Clinical Biochemistry, Vol. 38, No. 12, 2005, pp. 1103-1111. doi:10.1016/j.clinbiochem.2005.08.008

[20] M. Kosecik, O. Erel, E. Sevinc and S. Selek, "Increased Oxidative Stress in Children Exposed to Passive Smoking," International Journal of Cardiology, Vol. 100, No. 1, 2005, pp. 61-64. doi:10.1016/j.ijcard.2004.05.069

[21] J. K. Yao and R. Reddy, "Oxidative Stress in Schizophrenia: Pathogenetic and Therapeutic İmplications," Antioxidants \& Redox Signaling, Vol. 15, No. 7, 2011, pp. 1999-2002. doi:10.1089/ars.2010.3646

[22] A. Hoffer, H. Osmond and J. Smythies, "Schizophrenia: A New Approach," Journal of Mental Science, Vol. 100, No. 418, 1954, pp. 29-45.

[23] M. Padurariu, A. Ciobica, L. Hritcu, B. Stoica, W. Bild and C. Stefanescu, "Changes of Some Oxidative Stress Markers in the Serum of Patients with Mild Cognitive İmpairment and Alzheimer's Disease," Neuroscience Letters, Vol. 469, No. 1, 2010, pp. 6-10. doi:10.1016/j.neulet.2009.11.033

[24] M. Padurariu, A. Ciobica, I. Dobrin and C. Stefanescu, "Evaluation of Antioxidant Enzymes Activities and lipid Peroxidation in Schizophrenic Patients Treated with Typical and Atypical Antipsychotics," Neuroscience Letters, Vol. 479, No. 3, 2010, pp. 317-320. doi:10.1016/j.neulet.2010.05.088

[25] C. Jennewein, N. Tran, P. Paulus, P. Ellinghaus, J. A. Eble and K. Zacharowski, "Novel Aspects of Fibrin(Ogen) Fragments during Inflammation," Molecular Medicine, Vol. 17, No. 5-6, 2011, pp. 568-573. doi:10.2119/molmed.2010.00146

[26] B. E. Leonard, M. Schwarz and A. M. Myint, "The Metabolic Syndrome in Schizophrenia: Is Inflammation a Contributing Cause?" Journal of Psychopharmacology, Vol. 26, No. 5, 2012, pp. 33-41. doi: $10.1177 / 0269881111431622$

[27] S. R. Terlecky, L. J. Terlecky and C. R. Giordano, "Peroxisomes, Oxidative Stress, and Inflammation," World Journal of Biological Chemistry, Vol. 3, No. 5, 2012, pp. 93-97. doi:10.4331/wjbc.v3.i5.93

[28] A. Dietrich-Muszalska, J. Malinowska, B. Olas, R. Głowacki, E. Bald, B. Wachowicz and J. Rabe-Jabłońska, "The Oxidative Stress May Be Induced by the Elevated Homocysteine in Schizophrenic Patients," Neurochemical Research, Vol. 37, No. 5, 2012, pp. 1057-1062. doi:10.1007/s11064-012-0707-3

[29] J. K. Yao and M. S. Keshavan, "Antioxidants, Redox Signaling, and Pathophysiology in Schizophrenia: An İntegrative View," Antioxidants \& Redox Signaling, Vol.
15 , No. 7, 2011, pp. 2011-2035. doi:10.1089/ars.2010.3603

[30] G. Dadheech, S. Mishra, S. Gautam and P. Sharma, "Evaluation of Antioxidant Deficit in Schizophrenia," Indian Journal of Psychiatry, Vol. 50, No. 1, 2008, pp. 16-20. doi:10.4103/0019-5545.39753

[31] M. Raffa, A. Mechri, L.B. Othman, C. Fendri, L. Gaha and A. Kerkeni, "Decreased Glutathione Levels and Antioxidant Enzyme Activities in Untreated and treated Schizophrenic Patients," Progress in Neuro-Psychopharmacology and Biological Psychiatry, Vol. 33, No. 7, 2009, pp. 1178-1183. doi:10.1016/j.pnpbp.2009.06.018

[32] O. P. Singh, I. Chakraborty, A. Dasgupta and S. Datta, “A Comparative Study of Oxidative Stress and Interrelationship of Important Antioxidants in Haloperidol and Olanzapine Treated Patients Suffering from Schizophrenia," Indian Journal of Psychiatry, Vol. 50, No. 3, 2008, pp. 171-176. doi:10.4103/0019-5545.43627

[33] S. J. Wood, M. Yücel, C. Pantelis and M. Berk, "Neurobiology of Schizophrenia Spectrum Disorders: The Role of Oxidative Stress," Annals Academy of Medicine Singapore, Vol. 38, No. 5, 2009, pp. 396-406.

[34] G. Dakhale, S. Khanzode, S. Khanzode, et al., "Oxidative Damage and Schizophrenia: The Potential Benefit by Atypical Antipsychotics," Neuropsychobiology, Vol. 49, No. 4, 2004, pp. 205-209. doi:10.1159/000077368

[35] M. Kuloglu, B. Ustundag, M. Atmaca, et al., "Lipid Peroxidation and Antioxidant Enzyme Levels in Patients with Schizophrenia and Bipolar Disorder," Cell Biochemistry and Function, Vol. 20, No. 2, 2002, pp. 171-175. doi:10.1002/cbf.940

[36] M. Kunz, C. S. Gama, A. C. Andreazza, et al., "Elevated Serum Superoxide Dismutase and Thiobarbituric Acid Reactive Substances in Different Phases of Bipolar Disorder and in Schizophrenia," Progress in Neuro-Psychopharmacology and Biological Psychiatry, Vol. 32, No. 7, 2008, pp. 1677-1681. doi:10.1016/j.pnpbp.2008.07.001

[37] M. S. Rukmini, B. D’Souza and V. D’Souza, “Superoxide Dismutase and Catalase Activities and Their Correlation with Malondialdehyde in Schizophrenic Patients," Indian Journal of Clinical Biochemistry, Vol. 19, No. 2, 2004, pp. 114-118. doi:10.1007/BF02894268

[38] R. Reddy, M. P. Sahebarao, S. Mukherjee and J. N. Murthy, "Enzymes of the Antioxidant System in Chronic Schizophrenic Patients," Biological Psychiatry, Vol. 30, No. 4, 1991, pp. 409-412. doi:10.1016/0006-3223(91)90298-Z

[39] J. W. Newcomer, "Metabolic Syndrome and Mental Illness," American Journal of Managed Care, Vol. 13, No. 7, 2007, pp. S170-S177.

[40] S. Selek, N. Cosar, A. Kocyigit, et al., "PON1 Activity and Total Oxidant Status in Patients with Active Pulmonary Tuberculosis," Clinical Biochemistry, Vol. 41, No. 3, 2008, pp. 140-144. doi:10.1016/j.clinbiochem.2007.11.018

[41] N. Yilmaz, O. Aydin, A. Yegin, A. Tiltak and E. Eren, "Increased Levels of Total Oxidant Status and Decreased Activity of Arylesterase in Migraineurs," Clinical Biochemistry, Vol. 44, No. 10-11, 2011, pp. 832-837. 
doi:10.1016/i.clinbiochem.2011.04.015

[42] T. Gaillard, S. Parthasarathy and K. Osei, "HDL Dysfunctionality (Paraoxonase) Is Worse in Nondiabetic, Postmenopausal African American than in White Women," Diabetes Care, 2011, Vol. 34, No. 2, p. e19. doi:10.2337/dc10-1189

[43] K. N. Gan, A. Smolen, H. W. Eckerson and B. N. La Du, "Purification of Human Serum Paraoxonase/Arylesterase. Evidence for One Esterase Catalysing Both Activities," Drug Metabolism and Disposition, Vol. 19, No. 1, 1991, pp. 100-106.

[44] B. Mackness, M. I Mackness, S. Arrol, W. Turkie and P. N. Durrington, "Effect of the Human Serum Paraoxonase 55 and 192 Genetic Polymorphisms on the Protection by High Density Lipoprotein against Low Density Lipoprotein Oxidative Modification," FEBS Letters, Vol. 423, No. 1, 1998, pp. 57-60. doi:10.1016/S0014-5793(98)00064-7

[45] E. Boes, S. Coassin, B. Kollerits, I. M. Heid and F. Kronenberg, "Genetic-Epidemiological Evidence on Genes Associated with HDL Cholesterol Levels: A Systematic In-Depth Review," Experimental Gerontology, Vol. 44, No. 3, 2009, pp. 136-160. doi:10.1016/j.exger.2008.11.003

[46] S. Imaizumi, M. Navab, C. Morgantini, et al., "Dysfunctional High-Density Lipoprotein and the Potential of Apolipoprotein A-1 Mimetic Peptides to Normalize the Composition and Function of Lipoproteins," Circulation Journal, Vol. 75, No. 7, 2011, pp. 1533-1538. doi:10.1253/circj.CJ-11-0460
[47] N. Yilmaz, "Relationship between Paraoxonase and Homocysteine: Crossroads of Oxidative Diseases," Archives of Medical Science, Vol. 8, No. 1, 2012, pp. 138-153.

[48] N. Yilmaz, A. Yegin and G. Aykal, "New Noninvasive Modalities in Coronary Angiography-Diagnostic Values of New Biomarkers for Cardiovascular Disease," In: B. Baskot, Ed., Coronary Angiography-Advances in Noninvasive Imaging Approach for Evaluation of Coronary Artery Disease, InTech, Morn Hill, 2011, pp. 274-275.

[49] E. Eren, A. Yeğin, N. Yilmaz and H. Herken, "Serum Total Homocystein, Folate and Vitamin B12 Levels and Their Correlation with Antipsychotic Drug Doses in Adult Male Patients with Chronic Schizophrenia," Clinical Laboratory, Vol. 56, No. 11-12, 2010, pp. 513-518.

[50] C. U. Pae, I. H. Paik, C. Lee, S. J. Lee, J. J. Kim and C. U. Lee, "Decreased Plasma Antioxidants in Schizophrenia," Neuropsychobiology, Vol. 50, No. 1, 2004, pp. 54-56. doi:10.1159/000077942

[51] O. Virit, A. Altindag, M. Yumru, et al., "A Defect in the Antioxidant Defense System in Schizophrenia," Neuropsychobiology, Vol. 60, No. 2, 2009, pp. 87-93. doi:10.1159/000239684

[52] J. K. Yao, R. D. Reddy and D. P. van Kammen, “Abnormal Age-Related Changes of Plasma Antioxidant Proteins in Schizophrenia," Psychiatry Research, Vol. 97, No. 2, 2000, pp. 137-151. doi:10.1016/S0165-1781(00)00230-4 